\title{
P-0440 - Vitamin D status connect insulin resistance and bone turnover in Chinese non-osteoporosis patients with type 2 diabetes
}

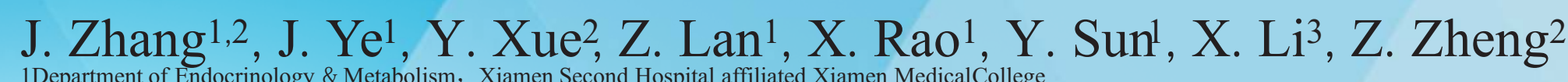

1Department of Endocrinology \& Metabolism, Xiamen Second Hospital affiliated Xiamen MedicalCollege
2Department of Endocrinology \& Metabolism Nanfang Hospital affiliated Southern Medical University

3Department of Endocrinology, The Second Hospital of Shanxi Medical University, Taiyuan 030001, China

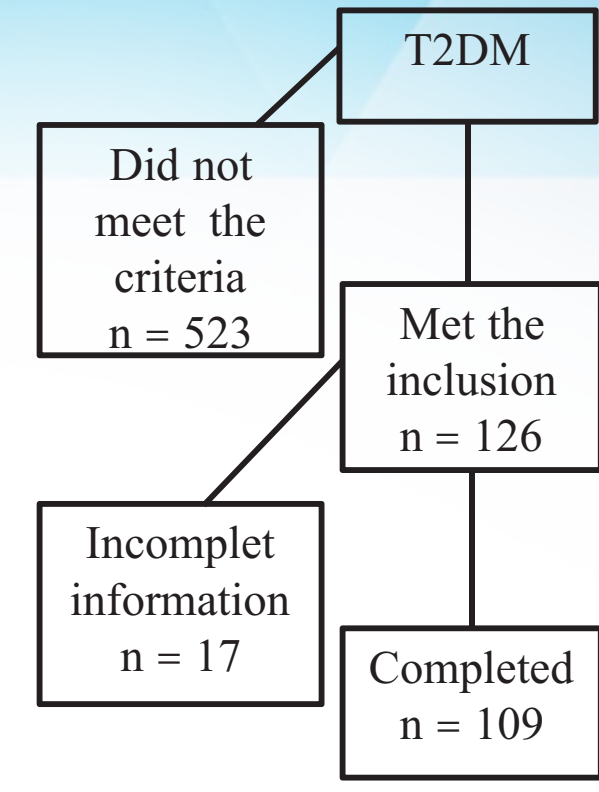

Fig1. The enrollment flow-chart

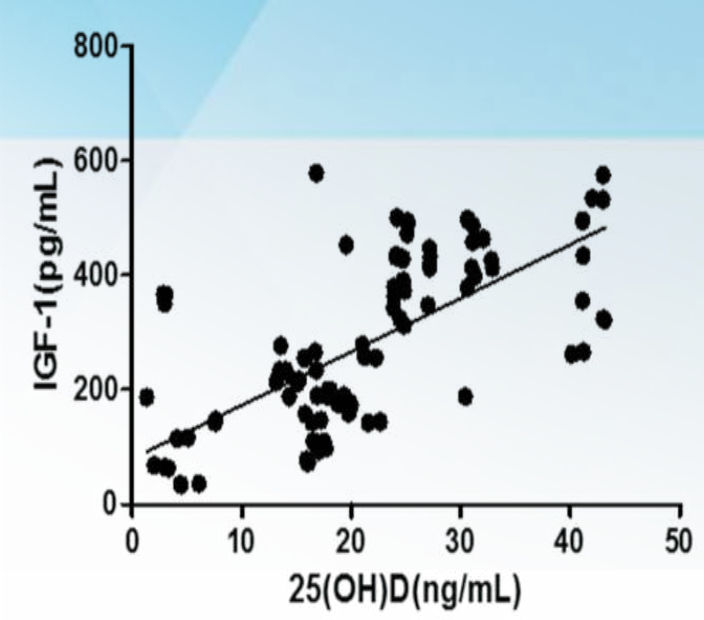

Fig2. The relationship between $25(\mathrm{OH}) \mathrm{D}$ and IGF-1 $(r=0.664$ $p=0.000)$

Background Vitamin D status was found closely related to blood glucose and bone metabolism in patients with type 2 diabetes (T2DM). Insufficiency and deficiency of vitamin $\mathrm{D}$ is related to development of type 2 diabetes. Vitamin D affected bone metabolism and bone density and resulted in osteopenia and osteoporosis. Insulin resistance raised the risk of osteoporosis. Based on the negative relationship between 25(OH)D concentrations and insulin resistance found in our research before, we boldly hypothesise that vitamin D status connects insulin resistance and bone turnover in non-osteoporosis patients with T2DM. Information is limited about the association between vitamin D status, insulin resistance and bone turnover in Chinese non-osteoporosis patients with T2DM.

Aim This study explored the relationship between serum $25(\mathrm{OH}) \mathrm{D}$ concentrations, bone turnover and insulin resistance in Chinese non-osteoporosis T2DM patients. Method This trial included 109 non-osteoporosis patients with T2DM. The enrollment flow-chart is showed in Fig1 . The parameters below were collected: fasting blood glucose (FBG), 25(OH)D, fasting blood insulin (FINS), serum creatinine $(\mathrm{SCr})$, glycosylated hemoglobin (HbA1c), calcium $(\mathrm{Ca})$, phosphorus $(\mathrm{P})$, parathyroid hormone (iPTH), insulin-like growth factor-1 (IGF-1), bone alkaline phosphatase (BALP), glomerular filtration rate (eGFR), body mass index (BMI), homeostasis model assessment of insulin resistance (HOMA-IR) and calcium-phosphorus product were performed. SPSS 19.0 software was used for statistical analysis. Multiple linear regression analysis was used to examine the associations below: (1)association beween serum $25(\mathrm{OH}) \mathrm{D}$ concentrations and HOMA-IR, (2) association beween serum $25(\mathrm{OH}) \mathrm{D}$ concentration and bone metabolism indece (calcium, phosphorus, BALP and IGF-1), $25(\mathrm{OH}) \mathrm{D}$, (3)association beween insulin resistance and bone metabolism indece (calcium, phosphorus, BALP and IGF-1), HOMA-IR, a $\mathrm{p}$ value $<0.05$ was considered significant.

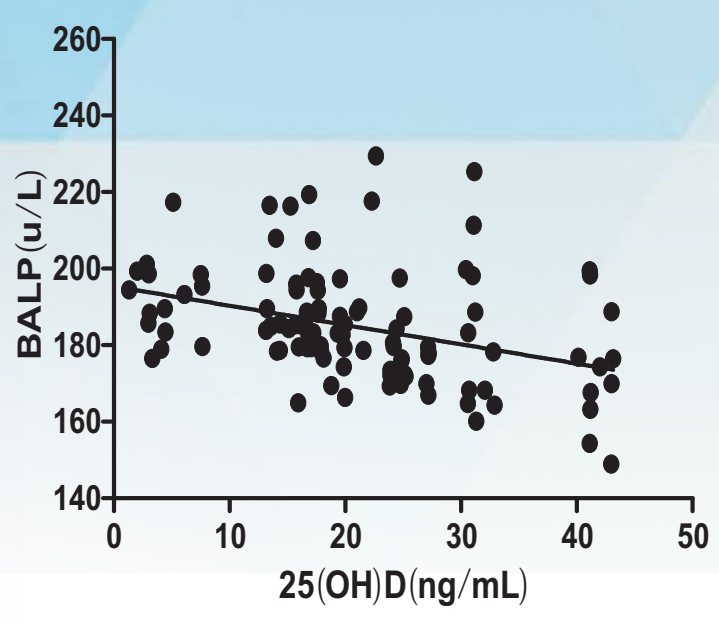

Fig3. The relationship between $25(\mathrm{OH}) \mathrm{D}$ and BALP $(r=-0.288$, $p=0.002)$

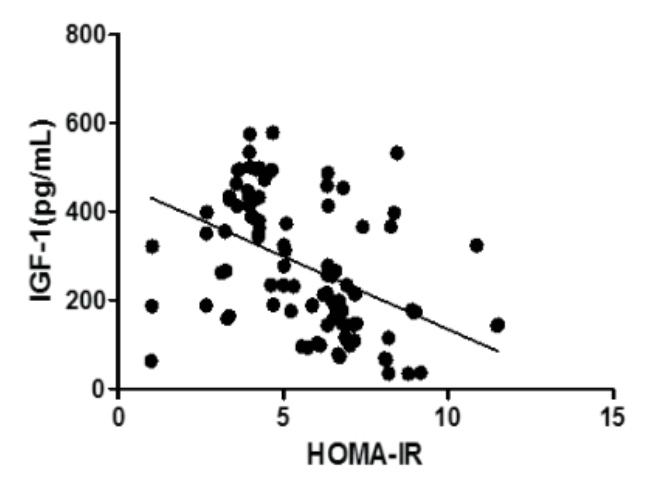

Fig5. The relationship between HOMA-IR and IGF-1 $(r=-0.460, p=0.000)$

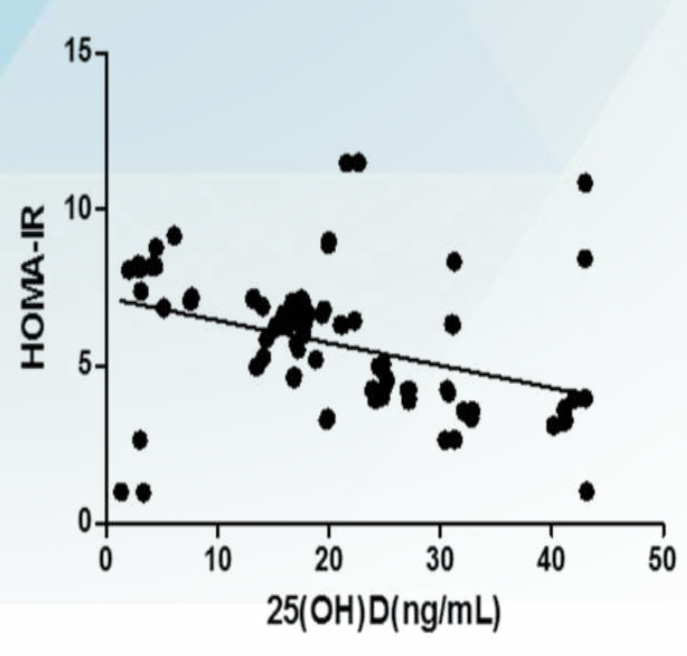
$25(\mathrm{OH}) \mathrm{D}$ and HOMA-IR $(r=-0.364, \quad p=0.000)$
Fig4. The relationship between

Results The average serum $25(\mathrm{OH}) \mathrm{D}$ value was $21.10 \pm 10.39 \mathrm{ng} / \mathrm{mL}$, below the sufficiency cutoff value of $30 \mathrm{ng} / \mathrm{mL}$. There was a positive correlation between 25(OH)D and IGF-1 ( $\beta=0.581, p=0.000)$, a negative correlation between $25(\mathrm{OH}) \mathrm{D}$ and BALP $(\beta=-0.182, p$ $=0.021)$ adjusted by age, BMI, eGFR. There was a negative correlation between 25(OH)D and HOMA-IR ( $\beta$ $=-0.349, p=0.000$ ) adjusted by age, BMI, eGFR Vitamin D status was a predictor of IGF-1 ( $\beta=0.581, p$ $=0.000)$, and BALP $(\beta=-0.182, p=0.021)$, as the dependent variable, but not eGFR, BMI, age, as independent variables. HOMA-IR were negatively correlated to IGF-1 $(\beta=-0.376, p=0.000)$, and positively correlated to BALP ( $\beta=0.232, p=0.013$ ), adjusted by age, eGFR , BMI. The Pearson corelation between 25(OH)D and IGF-1, 25(OH)D and BALP, 25(OH)D and HOMA-IR, HOMA-IR and IGF-1, HOMA-IR and BALP are showed in Tab1-5 respectively. Conclusion We speculate that $25(\mathrm{OH}) \mathrm{D}$ concentrations may interact with HOMA-IR to influence insulin resistance, thereby affecting bone turnover and eventually increasing the risk of osteoporosis in T2DM patients. 